\title{
A curcumin-loaded liquid crystal precursor mucoadhesive system for the treatment of vaginal candidiasis
}

This article was published in the following Dove Press journal:

International Journal of Nanomedicine

30 July 2015

Number of times this article has been viewed

\author{
Rafael Salmazi \\ Giovana Calixto \\ Jéssica Bernegossi \\ Matheus Aparecido dos \\ Santos Ramos \\ Taís Maria Bauab \\ Marlus Chorilli \\ School of Pharmaceutical Sciences, \\ UNESP - Sao Paulo State University, \\ Campus Araraquara, Department of \\ Drugs and Medicines, Araraquara, Sao \\ Paulo, Brazil
}

\begin{abstract}
Women often develop vaginal infections that are caused primarily by organisms of the genus Candida. The current treatments of vaginal candidiasis usually involve azole-based antifungals, though fungal resistance to these compounds has become prevalent. Therefore, much attention has been given to molecules with antifungal properties from natural sources, such as curcumin (CUR). However, CUR has poor solubility in aqueous solvents and poor oral bioavailability. This study attempted to overcome this problem by developing, characterizing, and evaluating the in vitro antifungal action of a CUR-loaded liquid crystal precursor mucoadhesive system (LCPM) for vaginal administration. A low-viscosity LCPM (F) consisting of 40\% wt/wt polyoxpropylene(5)-polyoxyethylene-(20)-cetyl alcohol, 50\% wt/wt oleic acid, and 10\% wt/wt chitosan dispersion at $0.5 \%$ with the addition of $16 \%$ poloxamer 407 was developed to take advantage of the lyotropic phase behavior of this formulation. Notably, F could transform into liquid crystal systems when diluted with artificial vaginal mucus at ratios of 1:3 and 1:1 (wt/wt), resulting in the formation of F30 and F100, respectively. Polarized light microscopy and rheological studies revealed that $\mathrm{F}$ behaved like an isotropic formulation, whereas F30 and F100 behaved like an anisotropic liquid crystalline system (LCS). Moreover, F30 and F100 presented higher mucoadhesion to porcine vaginal mucosa than $\mathrm{F}$. The analysis of the in vitro activity against Candida albicans revealed that CUR-loaded $\mathrm{F}$ was more potent against standard and clinical strains compared with a CUR solution. Therefore, the vaginal administration of CUR-loaded LCPMs represents a promising platform for the treatment of vaginal candidiasis.
\end{abstract}

Keywords: nanostructured drug delivery systems, liquid crystalline systems, mucoadhesive polymers, vaginal administration, Candida albicans

\section{Introduction}

Gynecological diseases affect women of all ages due to several factors, such as immune deficiency, poor hygiene, genetic conditions, and sexually transmitted diseases. These diseases can be caused by bacteria, protozoa, viruses, and fungi, though the most common infection is vaginal candidiasis, which is caused primarily by Candida albicans (C. albicans) but also by other Candida species. ${ }^{1,2}$

These opportunistic fungi strategically install themselves in the vaginal mucosa, frequently surviving attempted attacks by the immune system. Additional risk factors, such as recent antibiotic use, pregnancy, diabetes mellitus, oral contraceptives, and inadequate therapy, can further increase the incidence of the disease. ${ }^{3}$

The current treatment of candidiasis involves the administration of azole-derived drugs such as fluconazole, ketoconazole, and miconazole. Nevertheless, the clinical presentation of less susceptible strains leads to the failure of these treatments, increasing the recurrence of this disease. ${ }^{4}$ 
Thus, much emphasis has been placed on the discovery of new molecules from natural sources that can act effectively against various classes of Candida. ${ }^{1}$ Among these molecules, curcumin (CUR) stands out as a promising natural antifungal drug.

CUR, an intensely yellow pigment isolated from the rhizomes of the Curcuma longa plant, is a constituent of the spice turmeric and is used as a cooking spice, flavoring agent, and colorant. ${ }^{5}$

Furthermore, this natural drug has been shown to have antioxidant, anti-inflammatory, antiproliferative, anticancer, antiangiogenic, and antidiabetic properties in addition to its antifungal properties. ${ }^{6}$

Dovigo et $\mathrm{al}^{7}$ studied the use of CUR in association with a light-emitting diode for the inactivation of C. albicans isolated from patients with oral candidiasis. The results showed that there was a statistically significant reduction in the viability of $C$. albicans after the photodynamic therapy. ${ }^{7}$

Another study that used oral strains (no published studies have been conducted in vaginal strains) evaluated the inhibitory effects of CUR on the adhesion of Candida species isolated from patients with acquired immunodeficiency syndrome to human buccal epithelial cells in vitro. CUR dramatically inhibited the adhesion of Candida species, indicating that it is also a promising drug for immunocompromised patients. ${ }^{8}$

Nevertheless, the optimum performance of CUR is threatened by its poor solubility in aqueous solvents, which results in its poor oral bioavailability. $5,7,9$

To overcome these disadvantages, drug delivery nanostructure systems have emerged as an excellent alternative method for drug administration because these systems can enhance the solubility of drug, thus improving its bioavailability. ${ }^{10-13}$

Among these systems, liquid crystal (LC) precursor systems can be highlighted because they can incorporate water from the vaginal mucus to promote structural changes in the system, resulting in a liquid crystalline phase and thereby optimizing the efficiency of CUR. ${ }^{14,15}$

LCs belong to a state of matter with the properties of a crystalline solid and an isotropic liquid. In other words, the liquid crystalline phase is structurally similar to a crystalline solid, but with the disorder of a liquid, as demonstrated by the ease with which LC flow. ${ }^{16-18}$

LC can be formed by mixing oil, water, and a surfactant such as polyoxypropylene-(5)-polyoxyethylene-(20)-cetyl alcohol (PPG-5-CETETH-20), a nonionic surfactant that can form different liquid crystalline structures without requiring the use of a cosurfactant due to the absence of repulsive electrostatic interactions. This allows for the close packing of this surfactant in self-assembled structures. ${ }^{19}$

The contact time between the formulation and the vaginal mucosa can be optimized by using mucoadhesive polymer dispersions such as chitosan and poloxamer $(\mathrm{PO})$ as the aqueous phase of the liquid crystal precursor mucoadhesive system. This strategy is aimed at decreasing the repeated administration of the drug and overcoming the vaginal self-cleansing action that limits the formulation's residence time. ${ }^{20-22}$

$\mathrm{PO}$ is a polyoxyethylene copolymer that undergoes in situ gelling after exposure to an external stimulus. When the temperature is increased, the polymer aggregates into micelles due to the dehydration of the hydrophobic block of PO, which is followed by a transition from the sol phase to gel. ${ }^{23}$ Thus, the mucoadhesive mechanism of $\mathrm{PO}$ can be explained by its rheological properties, which decrease the mucociliary clearance and increase the contact time with the vaginal mucosa. ${ }^{24}$

Chitosan is a derivative of the polysaccharide chitin ( $\beta$-copolymer-(1-4)-D-glucosamine and $\beta$-(1-4)- $N$-acetyl-Dglucosamine) that is found abundantly in nature, especially in shell crustaceans. Owing to its cationic character, this polymer has aroused much interest for applications in mucoadhesive formulations because the mucoadhesion occurs via electrostatic interactions of the amino groups of chitosan with the sialic groups of mucin in the mucus layer. ${ }^{24,25}$

The conventional therapeutic modalities for the treatment of vaginal candidiasis are unfavorable because they cause recurrence of the disease. Therefore, the aim of this study was to develop a CUR-loaded liquid crystal precursor mucoadhesive system (LCPM) composed of PPG-5CETETH-20, oleic acid, and a chitosan dispersion with the addition of $\mathrm{PO}$, and to characterize this system using polarized light microscopy (PLM), rheological studies, and mucoadhesion assays. Moreover, we evaluated the in vitro antifungal action of the system in order to offer a promising and unexplored nanotechnology platform for the treatment of vaginal candidiasis.

\section{Materials and methods Materials}

PPG-5-CETETH-20 (Procetyl ${ }^{\circledR}$ AWS) was purchased from Croda (Campinas, Sao Paulo, Brazil). Oleic acid was purchased from Synth (Diadema, Sao Paulo, Brazil). Mucin from porcine stomach type II, CUR, PO 407, and low-molecular-weight chitosan were purchased from Sigma Aldrich (Steinheim, North Rhine-Westphalia, Germany). 
Amphotericin and Fluconazole were purchased from DEG (Sao Paulo, Sao Paulo, Brazil). The high-purity water was prepared with a Millipore Milli-Q Plus purification system, and its resistivity was $18.2 \mathrm{M} \Omega \cdot \mathrm{cm}$.

\section{Ternary phase diagram construction and preparation of LC precursor formulations}

The ternary phase diagram construction was performed by weighing and mixing different combinations of PPG-5CETETH-20 as the surfactant, oleic acid as the oil phase, and the chitosan dispersion at $0.5 \%$ as the aqueous phase (wt/wt) at room temperature $\left(25^{\circ} \mathrm{C} \pm 0.5^{\circ} \mathrm{C}\right)$. Next, $16 \%$ PO was added to each formulation. Each final formulation had a $\mathrm{pH}$ of 5.5.

Based on the structures identified by PLM described below, the ternary phase diagram was constructed using Sigma Plot Software.

The formulation F was selected as the LC precursor. CUR was added to $\mathrm{F}$, resulting in the formulation $\mathrm{FC}$.

$\mathrm{F}$ and $\mathrm{FC}$ were diluted with artificial vaginal mucus $(\mathrm{AVM})$ in a 1:3 (wt/wt) ratio to generate $\mathrm{F} 30$ and $\mathrm{FC} 30$ and a 1:1 (wt/wt) ratio to generate F100 and FC100, which were then used to evaluate the in situ phase behavior of the formulations. A volume of $1 \mathrm{~L}$ of AVM was prepared by mixing $3.51 \mathrm{~g}$ of NaCl, $1.40 \mathrm{~g}$ of $\mathrm{KOH}, 0.222 \mathrm{~g}$ of $\mathrm{Ca}(\mathrm{OH})_{2}$, $0.018 \mathrm{~g}$ of bovine serum albumin, $2.00 \mathrm{~g}$ of lactic acid, $1.00 \mathrm{~g}$ of acetic acid, $0.16 \mathrm{~g}$ of glycerol, $0.4 \mathrm{~g}$ of urea, $5.0 \mathrm{~g}$ of glucose, and $15 \mathrm{~g}$ of mucin. After complete mixing, the $\mathrm{pH}$ was adjusted to 4.2 using $0.1 \% \mathrm{HCl}^{26,27}$

\section{Structural characterization of selected formulations PLM}

A drop of each formulation was placed on a glass slide, covered with a coverslip and examined under polarized light at room temperature $\left(25^{\circ} \mathrm{C} \pm 0.5^{\circ} \mathrm{C}\right)$ using an Olympus BX41 polarized light microscope coupled with a Q-Color3 camera (Olympus America, Inc, Center Valley, PA, USA). The magnification was $20 \times .{ }^{15}$

\section{In vitro evaluation of the mucoadhesive force}

The mucoadhesive force between the porcine vaginal mucosa and the formulations was assessed in a detachment test using a TA-XT plus texture analyzer (Stable Micro Systems, Surrey, UK). Freshly excised pig vaginal mucosae were obtained from a local slaughterhouse, cleaned, and frozen at $-30^{\circ} \mathrm{C}$ until the day of the experiment. After defrosting, a $2 \mathrm{~mm}$ thick section was taken from the inner part of the surface of the vaginal mucosa and fitted onto the mucoadhesion test rig. Then, $50 \mu \mathrm{L}$ of simulated vaginal mucus was applied to the surface of the tissue before the experiment. ${ }^{28}$ The formulations were packed into shallow cylindrical vessels, and the analytical probe was lowered to begin the test. The probe, which contained the mucosa, moved at a constant speed $\left(1 \mathrm{~mm} \mathrm{~s}^{-1}\right)$ on the surface of the formulation. The mucosa and the formulation were kept in contact for 60 seconds, and no force was applied during this interval. After 60 seconds, the mucosa was drawn upward $\left(0.5 \mathrm{~mm} \mathrm{~s}^{-1}\right)$ until the contact between the surfaces was broken. The mucoadhesive force of the formulations was measured in the maximum detachment force as the resistance to the withdrawal of the probe, which reflects the mucoadhesion characteristics. Seven replicates were analyzed at $37^{\circ} \mathrm{C} \pm 0.5^{\circ} \mathrm{C} .^{21}$

\section{Rheological analysis}

The rheological measurements were performed at $37^{\circ} \mathrm{C} \pm 0.1^{\circ} \mathrm{C}$ in triplicate using a controlled-stress AR2000 rheometer (TA Instruments, New Castle, DE, USA) with cone plate geometry (40 mm diameter) and a sample gap of $52 \mu \mathrm{m}$. The samples of the formulations were carefully applied to the lower plate to minimize the sample shearing and were allowed to equilibrate for 3 minutes prior to analysis.

\section{Determination of flow properties}

The flow properties were determined using a controlled shear rate procedure ranging from 0.01 to $100 \mathrm{~s}^{-1}$ and back. Each stage lasted 120 seconds with an interval of 10 seconds between the curves. The consistency and flow indexes were determined from the power law described in Equation 1 for a quantitative analysis of flow behavior:

$$
\tau=\kappa \cdot \gamma^{\eta}
$$

where " $\tau$ " is the shear stress, " $\gamma$ " is the shear rate, " $\kappa$ " is the consistency index, and " $\eta$ " is the flow index. ${ }^{29}$

\section{Oscillatory analyses}

The oscillatory analyses were started by the conduction of a stress sweep in order to determine the viscoelastic region of the formulations. The stress sweep was carried out at a constant frequency of $1 \mathrm{~Hz}$ over the stress range of $0.1-10 \mathrm{~Pa}$. A constant shear stress of $1 \mathrm{~Pa}$ was selected to perform the frequency sweep over a range of $0.1-10 \mathrm{~Hz}$, which was within the previously determined linear viscoelastic region for all formulations. Thus, the storage $\left(\mathrm{G}^{\prime}\right)$ and loss $\left(\mathrm{G}^{\prime \prime}\right)$ moduli 
were recorded. The variation of the storage modulus $\left(G^{\prime}\right)$ at low frequencies in a log-log plot of $G^{\prime \prime}$ versus $\omega$ followed the power law described in Equation 2, given by:

$$
\mathrm{G}^{\prime}=S \cdot \omega^{\mathrm{n}}
$$

where $\mathrm{G}^{\prime}$ is the storage modulus, $S$ is the formulation strength, $\omega$ is the oscillation frequency, and $n$ is the viscoelastic exponent. ${ }^{29}$

\section{In vitro evaluation of antifungal activity} Fungal strains

Two strains of $C$. albicans were used, a standard strain (ATCC 18804, American Type Culture Collection) and a clinical strain (CAV 2), which was isolated from the vaginal area of a patient with a positive diagnosis of vulvovaginal candidiasis. Both strains are part of the mycological collection of clinical strains of microorganisms from the Laboratory of Microorganisms Physiology of the Department of Biological Sciences, Faculty of Pharmaceutical Sciences of Araraquara. Both yeast strains were maintained on Sabouraud Dextrose Broth (SDB) plus $20 \%$ glycerol and frozen at $-20^{\circ} \mathrm{C}$. For use, the strains were subcultured in $2 \mathrm{~mL}$ of SDB and incubated at $37^{\circ} \mathrm{C}$ for 48 hours. The use of the vulvovaginal strains for this study was approved by the local ethics committee.

\section{Preparation of fungal suspensions}

The grown yeasts were transferred to sterile phosphate-buffered saline and diluted to a $0.5 \mathrm{McF}$ arland standard $\left(10^{6} \mathrm{CFU} /\right.$ $\mathrm{mL}$ ), which was confirmed by a spectrophotometric reading at $620 \mathrm{~nm}$ and cell counting in a Neubauer chamber. Then, this fungal suspension was diluted to 1:1,000 to yield a suspension of $10^{3} \mathrm{CFU} / \mathrm{mL}$, which was used in the experiments. ${ }^{30}$

\section{Determination of the minimum inhibitory concentration (MIC)}

The evaluation of the antifungal activity and MIC determination was carried out using a microdilution technique according to the methodology described by document M27-A3 from the Clinical and Laboratory Standards Institute 2008, with modifications. ${ }^{31}$

A volume of $100 \mu \mathrm{L}$ of Roswell Park Memorial Institute (RPMI) culture medium 1640 at $\mathrm{pH} 7.0$ was added to each well of the microplate (96 wells). Next, $100 \mu \mathrm{L}$ of the CUR solution and FC was also added to each well. Serial dilutions were made over a range of 1,000 to $7.8 \mathrm{mg} / \mathrm{mL}$. Next, 100 $\mu \mathrm{L}$ of the fungal suspension was distributed in each well of the microplate.
Amphotericin B (16.0-0.06 $\mu \mathrm{g} / \mathrm{mL})$ and fluconazole $(512-1.0 \mathrm{mg} / \mathrm{mL})$ were used as positive controls. The medium, the yeast growth, the F formulation, and the sterility of the solvent were also controlled.

The microplates were incubated at $37^{\circ} \mathrm{C}$ for 48 hours. Then, $20 \mu \mathrm{L}$ of an aqueous solution of $2 \% 2,3,5$-triphenyltetrazolium chloride (TTC) was added, and the plates were incubated at $37^{\circ} \mathrm{C}$ for 3 hours. The absence of growth of the microorganisms maintained the colorless solutions in the wells, whereas red-colored solutions indicated the microorganism growth.

\section{Statistical analyses}

Statistical analyses were performed using standard methods. ANOVA was employed to calculate significance $(P<0.05)$.

\section{Results and discussion Ternary phase diagram construction and preparation of the LC precursor formulations}

A phase diagram was constructed to assess the combination of different proportions of the surfactant, oil phase, and aqueous phase used in making the formulations.

After the preparation of the various combinations, the resulting formulations were characterized visually as opaque liquid systems (OLS), transparent liquid systems (TLS), transparent viscous systems (TVS), and phase separations (PS). This resulted in the construction of the diagram shown in Figure 1.

Figure 1 shows that it was possible to obtain a transparent viscous system when the concentrations of the surfactant, the oil phase, and the aqueous phase were $10 \%-55 \%, 10 \%-50 \%$, and above $40 \%$, respectively. The transparent liquid system was formed in two regions of the diagram: the first region was formed when the concentrations of the surfactant, the oil phase, and the aqueous phase were greater than $55 \%, 5 \%-40 \%$, and below $35 \%$, respectively; the second region was formed when the concentrations of the surfactant, the oil phase, and the aqueous phase were $25 \%-45 \%, 45 \%-65 \%$, and $5 \%-30 \%$, respectively. The opaque liquid systems were obtained in the regions where the concentrations of the surfactant, the oil phase, and the aqueous phase were below $25 \%, 30 \%-75 \%$, and $15 \%-70 \%$, respectively. Phase separation occurred in a small proportion of the formulations, in which the concentrations of the surfactant, the oil phase, and the aqueous phase were below $30 \%, 65 \%-100 \%$, and below $30 \%$, respectively. 


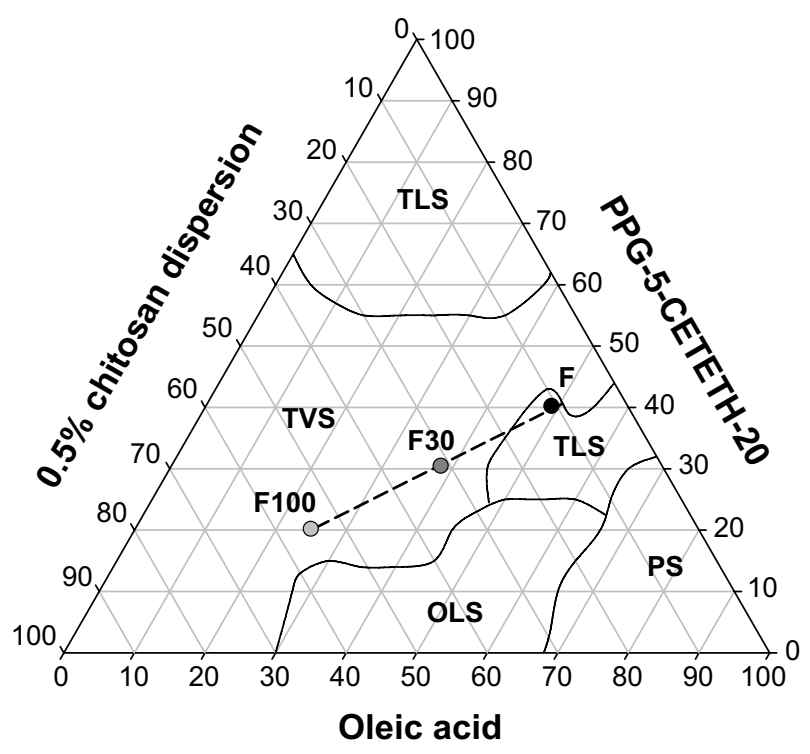

Figure I Ternary phase diagram of PPG-5-CETETH-20, oleic acid, and $0.5 \%$ chitosan dispersion associated with $16 \%$ poloxamer 407 .

Notes: $\mathrm{F}$ is the liquid crystal precursor system, $\mathrm{F} 30$ is a $3: \mathrm{I}$ (wt/wt) dilution of $\mathrm{F}$ containing $30 \%$ artificial vaginal mucus, and $\mathrm{FI} 00$ is a $\mathrm{I}: \mathrm{I}$ (wt/wt) dilution of $\mathrm{F}$ containing $100 \%$ artificial vaginal mucus.

Abbreviations: PS, phase separation; OLS, opaque liquid systems; TLS, transparent liquid systems; TVS, transparent viscous systems; PPG-5-CETETH-20, polyoxypropylene-(5)-polyoxyethylene-(20)-cetyl alcohol.

All of the transparent liquid systems formed were isotropic because they appeared dark when visualized under polarized light. In contrast, the transparent viscous systems were anisotropic because they showed various structures that are typical of liquid crystalline systems.

Based on the phase diagram, formulation $\mathrm{F}$, a liquid formulation composed of 40\% wt/wt PPG-5-CETETH-20, $50 \% \mathrm{wt} / \mathrm{wt}$ oleic acid, and $10 \% \mathrm{wt} / \mathrm{wt}$ chitosan dispersion at $0.5 \%$ with the addition of $16 \%$ PO 407 , was selected as the LC precursor system because its liquid phase facilitates its administration (eg, by syringe).

Moreover, $\mathrm{F}$ is located in a phase transition region. Thus, when diluted with vaginal mucus, the viscosity of $\mathrm{F}$ increased in situ. This can be observed in the dilution line shown in the diagram in Figure 1.

Carvalho et $\mathrm{al}^{34}$ developed a phase diagram consisting of oleic acid as the oil phase, PPG-5-CETETH-20 as the surfactant, and water as the aqueous phase. This also resulted in different regions. However, they have reported a lower region of transparent viscous systems, which may be due to the absence of mucoadhesive polymers such as chitosan and PO, which are present in the diagram of Figure 1. Such polymers may have interacted with the other components of the system, changing the rheological property of these systems, which led to an increase in the region of viscous systems of the diagram. ${ }^{21,29}$

\section{Structural characterization of selected formulations \\ PLM}

The photomicrographs obtained from the PLM of F, F30, and F100 are illustrated in Figure 2. The CUR-loaded formulations of F, F30, and F100 showed the same patterns as the unloaded formulations.

The F photomicrograph is represented by a dark field, which is a typical characteristic of microemulsions (MEs) that are clear, isotropic, and thermodynamically stable colloidal systems obtained spontaneously from surfactant-cosurfactant combinations. ${ }^{32}$

Malta crosses were observed in the photomicrograph of F30, suggesting that a lamellar liquid crystalline mesophase was formed with the incorporation of $30 \%$ AVM (wt/ $\mathrm{wt}){ }^{13,33}$

The F100 photomicrograph shows striated structures that may be the more organized structures that are typical of hexagonal liquid crystalline mesophase. ${ }^{33}$

Therefore, these results demonstrated that $\mathrm{F}$ behaved as a precursor of a LC system and was able to form a strong LC mesophase with the incorporation of AVM.

This transition can be attributed to an increase in the packing constraint in the hydrophilic core of PPG-5-CETETH-20, which reduced the interfacial curvature of the aggregate. Moreover, the subsequent hydration of the surfactant generated a great repulsive force between the head groups of the surfactant, increasing the distances between the lamellae. This resulted in the formation of different liquid crystalline structures. ${ }^{34}$

\section{In vitro evaluation of the mucoadhesive force}

The work performed by the mucoadhesive force of the unloaded and CUR-loaded formulations is illustrated in Figure 3.

The incorporation of 30\% AVM significantly increased the mucoadhesive work of both F and FC, which may have been due to changes in the rheological properties of these formulations, as F and FC were classified as TLS, while F30 and FC30 were classified as TVS.

Unexpectedly, the mucoadhesive force of F100 and FC100 decreased with the incorporation of $100 \%$ AVM. The more viscous formulations, such as the hexagonal formulations F100 and FC100, tended to be more mucoadhesive, as explained earlier. However, some studies have indicated that the formulations with very rigid structural matrixes, such as F100 and FC100, can prevent interactions between the formulation and the biological membrane proteins, thus decreasing the mucoadhesion values. ${ }^{34}$ 

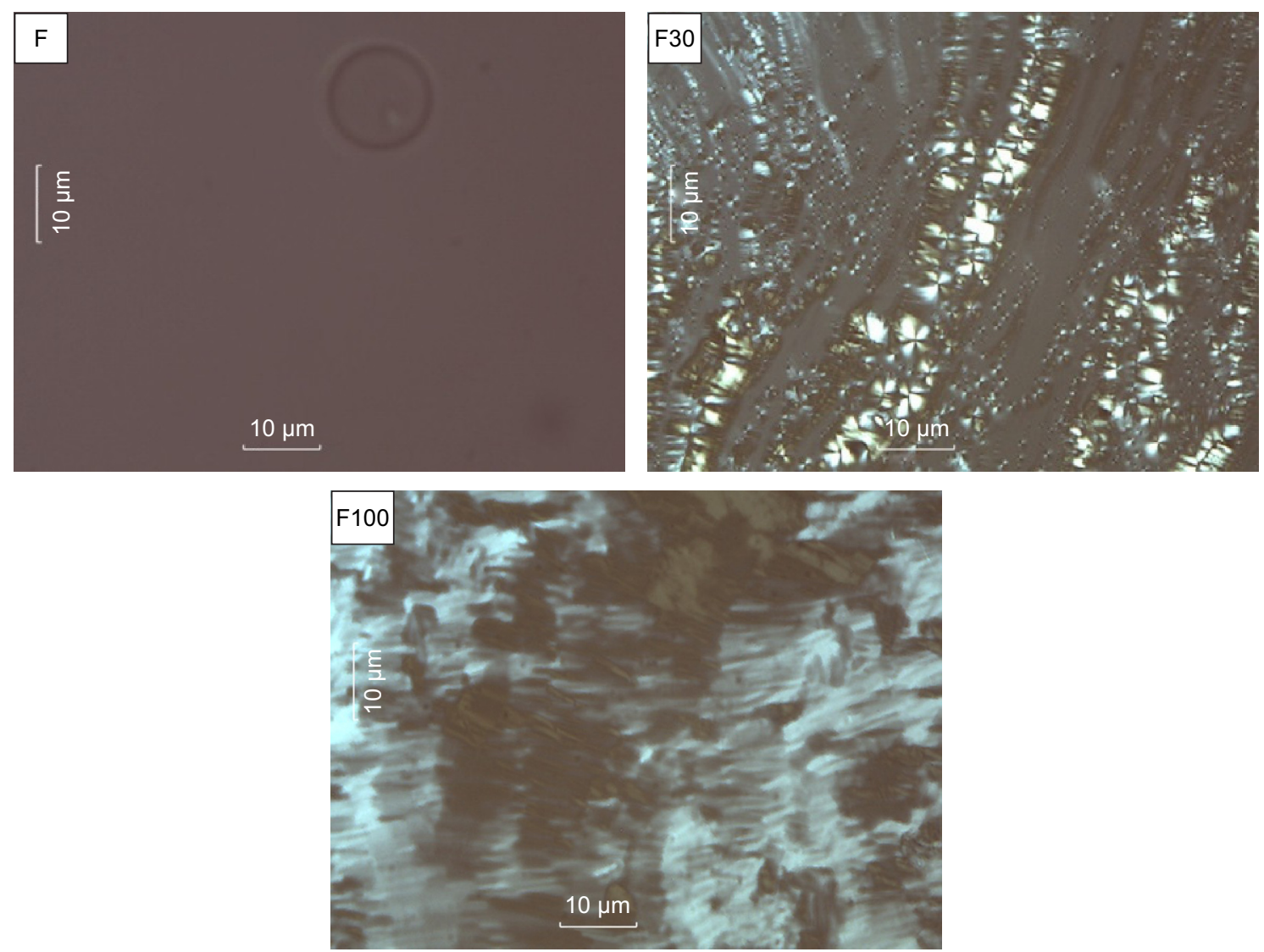

Figure 2 Photomicrographs of the F, F30, and FI00 formulations. Note: Magnification 20x.

In addition, the low mucoadhesion values of F100 and FC100 may have been due to the presence of a larger amount of mucin because these formulations contained 100\% AVM. The chitosan and PO polymers have a high affinity for mucin, which may have prevented the formation of a stronger bond

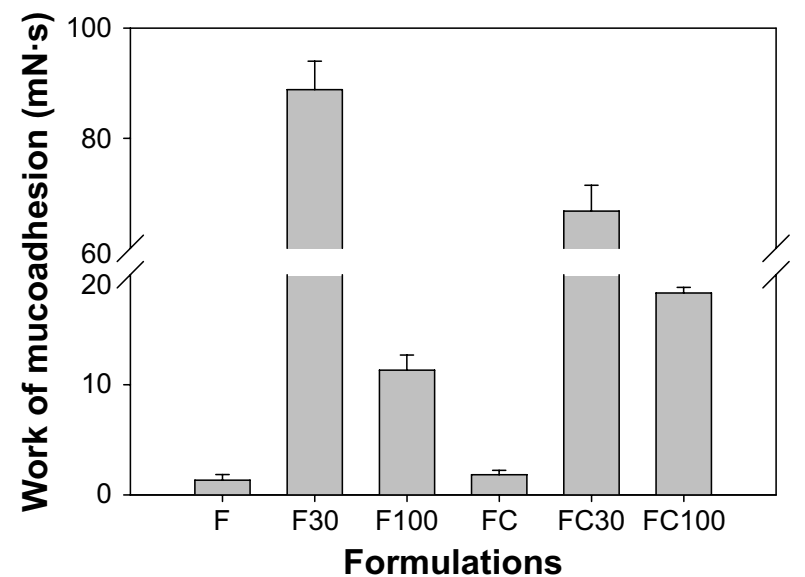

Figure 3 Mucoadhesion work (N.s) of the unloaded (F, F30, and FI00) and CURloaded (FC, $\mathrm{FC} 30$, and $\mathrm{FCl} 00$ ) formulations.

Notes: Each value represents the mean \pm standard deviation of at least seven replicates. The data were collected at $37^{\circ} \mathrm{C} \pm 0.5^{\circ} \mathrm{C}$.

Abbreviation: CUR, curcumin. between the F100 and FC100 formulations and the mucosa. This is because the interpenetration of the mucosal network by the polymer may be responsible for the mucoadhesive properties of the formulation. ${ }^{35}$

However, F100 and FC100 showed mucoadhesion values that were very similar to the formulation composed of $55 \%$ w/w PPG-5-CETETH-20, 30\% wt/wt oleic acid, and 15\% $\mathrm{wt} / \mathrm{wt}$ water diluted 1:1 (wt/wt) with artificial nasal mucus developed by Carvalho et al. ${ }^{15}$

Thus, this finding further demonstrates that incorporating mucoadhesive polymers such as chitosan and PO into the formulation in addition to increasing the viscosity also increases the mucoadhesive characteristics of the formulation. This may have conferred advantages on the formulations developed here because a higher mucoadhesion value may prolong the time of action and the absorption of the drug, thereby improving the clinical performance of the formulation.

\section{Rheological studies}

The relationships between the shear rate and the shear stress of all formulations are shown in the rheograms in Figure 4. 

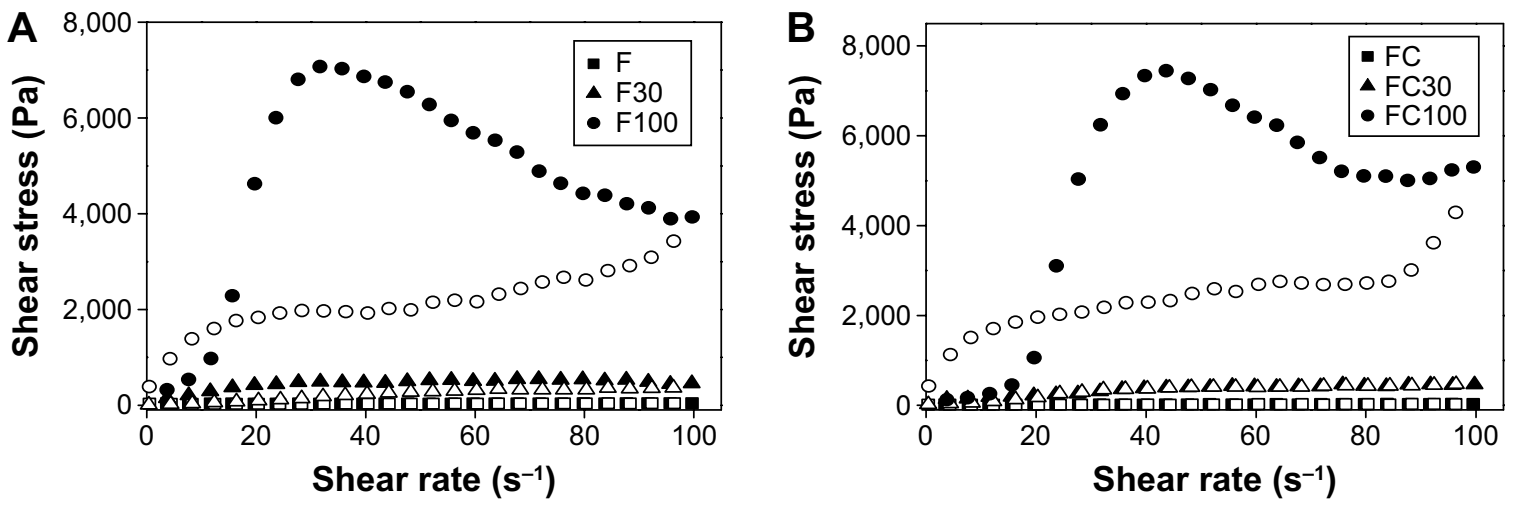

Figure 4 Flow rheograms of unloaded and CUR-loaded formulations.

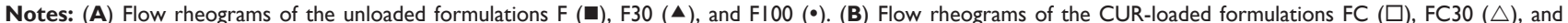
$\mathrm{FCI} 00(\mathrm{O})$. The closed symbols represent the up-curves, and the open symbols represent the down-curves. The standard deviations have been omitted for clarity; however, in all cases, the coefficients of variation of the triplicate analyses were less than $10 \%$. The data were collected at $37^{\circ} \mathrm{C} \pm 0.5^{\circ} \mathrm{C}$.

Abbreviation: CUR, curcumin.

The unloaded (F, F30, and F100) and CUR-loaded (FC, FC30, and FC100) formulations showed nonlinear relationships between the shear stress and the shear rate, exhibiting non-Newtonian flow characteristics. The data obtained by Equation 1 and shown in Table 1 indicated that all formulations exhibited shear thinning and pseudoplastic behavior because all the obtained $\eta$-values were less than unity $(\eta<1)$.

Pseudoplastic behavior is a desirable property for vaginal formulations because pseudoplastic formulations experience flow thinning under the application of a stress, which facilitates the administration of such a formulation via syringe. This thinning can be caused by a breakdown of the organized structures of the formulation in the flow direction. . $^{5,30,33}$

Rheological analyses of vaginal hydrophilic polymer gels and microemulsion-based gels for the vaginal delivery of clotrimazole have shown that these formulations also exhibit pseudoplastic behavior. This ensures their immediate flow after the application of stress, as is the case for the formulations developed in the present study. ${ }^{36}$

Table 1 also shows the $k$ values of all formulations. The $k$ value of F100 was increased relative to the $k$ values of $\mathrm{F}$ and $\mathrm{F} 30$; this was also the case for the $k$ value of $\mathrm{FC} 100$

Table I Consistency index $(k)$ and flow index $(\eta)$ of unloaded $(\mathrm{F}, \mathrm{F30}, \mathrm{FIO0})$ and curcumin-loaded (FC, FC30, FCI00) formulations

\begin{tabular}{lll}
\hline Formulations & $\boldsymbol{\eta}$ & $\mathbf{k}(\mathbf{P a} \cdot \mathbf{s})$ \\
\hline F & 0.20 & 33.2 \\
F30 & 0.23 & 189.2 \\
FI00 & 0.21 & $2,151.0$ \\
FC & 0.25 & 7.7 \\
FC30 & 0.41 & 73.6 \\
FC100 & 0.41 & 971.0 \\
\hline
\end{tabular}

compared with the $k$ values of FC and FC30. These results show the enhanced thickening of F100 and FC100.

From the rheograms in Figure 4, it was also observed that only F100 and FC100 presented notable thixotropic responses. This phenomenon demonstrated that these formulations had well-organized hexagonal structures after the incorporation of $100 \%$ AVM. Thus, there was a disruption of these structures when the stress was increased. When the stress was removed, these formulations required a longer time to return to their original organized hexagonal configuration. $^{21,29,32}$

All these results demonstrate that CUR did not alter the behavior of the formulations. However, the incorporation of AVM had a strong influence on the liquid crystalline structure in terms of the rheological properties of each formulation.

The oscillatory analyses of all formulations are shown in Figure 5, in which the storage modulus $\mathrm{G}^{\prime}$ and the loss modulus $\mathrm{G}^{\prime \prime}$ are plotted against the frequency.

$\mathrm{F}$ and $\mathrm{FC}$ were demonstrated to be more viscous than elastic $\left(\mathrm{G}^{\prime}<\mathrm{G}^{\prime \prime}\right)$. However, the formulations containing added AVM became elastic formulations $\left(G^{\prime}>G^{\prime \prime}\right)$, presenting gel-like behaviors with highly organized structures.

Furthermore, the data obtained by Equation 2 and shown in Table 2 indicated that the incorporation of AVM also increased the $S$ values and decreased the $n$ values of $\mathrm{F}$ and FC. This indicated that increased contacts were made between the networked structures of these formulations and the vaginal mucus layer.

It could also be observed that the $\mathrm{G}^{\prime}, \mathrm{S}$, and $\mathrm{n}$ values for F100 and FC100 decreased. This suggested that these formulations tended to transition to less organized phases, as seen in simple emulsified systems. ${ }^{34}$ Although the values of 

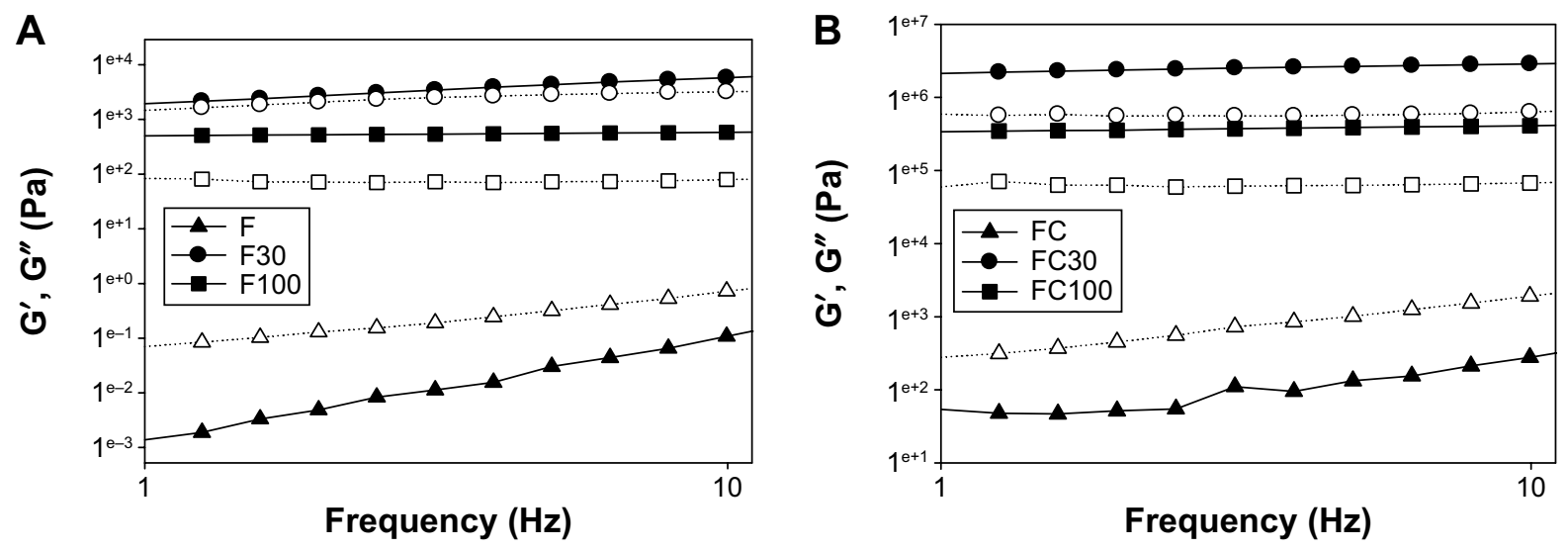

Figure 5 Frequency sweep profiles of unloaded and CUR-loaded formulations.

Notes: $(\mathbf{A})$ Frequency sweep profile of the storage modulus $G^{\prime}$ (closed symbols) and loss modulus $G^{\prime \prime}$ (opened symbols) of the unloaded formulations $F(\boldsymbol{\Delta})$, F30 ( $\bullet$ ), and

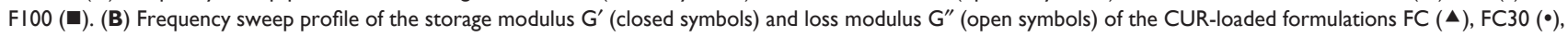
and FCI00 (ם). The SDs have been omitted for clarity; however, in all cases, the coefficients of variation of the triplicate analyses were less than I0\%. The data were collected at $37^{\circ} \mathrm{C} \pm 0.5^{\circ} \mathrm{C}$.

Abbreviations: CUR, curcumin; SD, standard deviation.

$\mathrm{G}^{\prime}, \mathrm{S}$, and $\mathrm{n}$ were the lowest for F100 and FC100, they were close to the values shown by bioadhesive hydrogels. ${ }^{28}$

Therefore, the present formulations are acceptable even at dilutions of $1: 1$, demonstrating that the in situ gelling properties of $\mathrm{F}$ and $\mathrm{FC}$ may potentially be related to the bioavailability of their loaded drugs: elastic formulations are retained for longer in the mucous membrane, which thereby increases the drug absorption time.

\section{In vitro evaluation of antifungal activity}

Table 3 shows the results obtained from the MIC analysis of the two strains of $C$. albicans. The MIC of free CUR against both strains was $1,000 \mu \mathrm{g} / \mathrm{mL}$. By contrast, the MIC of FC against C. albicans ATCC was $62.5 \mu \mathrm{g} / \mathrm{mL}$, and against C. albicans CAV2 (the clinical strain) was $500 \mu \mathrm{g} / \mathrm{mL}$.

This finding clearly demonstrates the crucial role of liquid crystalline formulations because the action of incorporated CUR was improved. Other studies have shown that liquid crystalline systems have an affinity for the lipids present in the fungal cell wall. This can increase the intracellular absorption of CUR by the fungi, resulting in increased antifungal activity. ${ }^{37,38}$

Table 2 Storage modulus $\left(\mathrm{G}^{\prime}\right)$, formulation strength $(S)$, and viscoelastic exponent $(n)$ of the unloaded and curcumin-loaded formulations

\begin{tabular}{llll}
\hline Formulations & $\mathbf{G}^{\prime}$ & $\mathbf{S}$ & $\mathbf{n}$ \\
\hline F & $\mathrm{I} .5 \mathrm{I}$ & $0.4 \mathrm{I}$ & 0.42 \\
F30 & $6,879.42$ & $2,910.1 \mathrm{I}$ & 0.28 \\
FI00 & 683.43 & 394.34 & 0.18 \\
FC & 1.85 & 0.25 & 0.59 \\
FC30 & $3,154.17$ & $2,105.23$ & 0.14 \\
FCI00 & 434.74 & 327.26 & 0.10 \\
\hline
\end{tabular}

Souza et $\mathrm{al}^{39}$ also developed and tested the antimicrobial activity of a liquid crystalline formulation incorporating poly (hexamethylene biguanide) hydrochloride (PHMB), a biocidal polymer, against Streptococcus mutans and C. albicans. Their results showed that the action of PHMB was improved by loading this drug into the liquid crystalline formulation, indicating that this formulation can be an alternative for the administration of PHMB.

The LCPM developed in the present study improved the mucoadhesion of the system to the vaginal mucosa. Moreover, this system promoted the antimicrobial activity of CUR against C. albicans. Thus, this formulation shows promise as a drug delivery system for the vaginal administration of CUR.

\section{Conclusion}

PLM and rheological analyses showed that a LCPM composed of 40\% wt/wt PPG-5-CETETH-20, 50\% wt/wt oleic acid, and $10 \% \mathrm{wt} / \mathrm{wt}$ chitosan dispersion at $0.5 \%$ containing added 16\% PO 407 showed in situ gelling properties caused by the addition of AVM. The addition of AVM caused this

Table 3 Minimum inhibitory concentration (MIC, $\mu \mathrm{g} / \mathrm{mL}$ ) of curcumin against Candida albicans strains ATCC 18804 and CAV 2

\begin{tabular}{lll}
\hline Samples & C. albicans ATCC I8804 & C. albicans CAV 2 \\
\hline Free CUR & $1,000 \mu \mathrm{g} / \mathrm{mL}$ & $\mathrm{I}, 000 \mu \mathrm{g} / \mathrm{mL}$ \\
FC & $62.5 \mu \mathrm{g} / \mathrm{mL}$ & $500 \mu \mathrm{g} / \mathrm{mL}$ \\
F & No inhibition & No inhibition \\
Amphotericin B & $0.50 \mu \mathrm{g} / \mathrm{mL}$ & $2.00 \mu \mathrm{g} / \mathrm{mL}$ \\
Fluconazole & $128 \mu \mathrm{gL}$ & Resistant \\
DMSO 20\% & No inhibition & No inhibition \\
\hline
\end{tabular}


precursor formulation to transform into an anisotropic liquid crystalline formulation with pseudoplasticity and increased viscosity. The mucoadhesion analyses showed that the inclusion of the polymers increased the mucoadhesive power of the formulation compared with the formulations without the polymers. Finally, the in vitro antifungal analysis against C. albicans revealed that the CUR-loaded formulation was sixteen times more potent against a standard strain and two times more potent against a clinical strain than free CUR. Therefore, the set of results demonstrated that this LCPM for the vaginal administration of CUR is a promising alternative for the treatment of vaginal candidiasis.

\section{Acknowledgments}

This work was financially supported by Fundação de Amparo à Pesquisa do Estado de São Paulo (FAPESP), Conselho Nacional de Desenvolvimento Científico e Tecnológico (CNPq), and Programa de Apoio ao Desenvolvimento Científico (PADC).

\section{Disclosure}

The authors report no conflicts of interest in this work.

\section{References}

1. da Silva PB, Ramos MA, Bonifácio BV, et al. Nanotechnological strategies for vaginal administration of drugs - a review. $J$ Biomed Nanotechnol. 2014;10(9):2218-2243.

2. Dou N, Li W, Zhou E, Wang C, Xiao Z, Zhou H. Risk factors for candida infection of the genital tract in the tropics. Afr Health Sci. 2015; 14(4):835-839.

3. Tsai PW, Chen YT, Hsu PC, Lan CY. Study of Candida albicans and its interactions with the host: a mini review. Bio Medicine. 2013; 3(1):51-64.

4. Machado-de-Sena RM, Corrêa L, Kato IT, et al. Photodynamic therapy has antifungal effect and reduces inflammatory signals in Candida albicans-induced murine vaginitis. Photodiagn Photodyn Ther. 2014; 11(3):275-282.

5. Dovigo LN, Pavarina AC, Ribeiro AP, et al. Investigation of the photodynamic effects of curcumin against Candida albicans. Photochem Photobiol. 2011;87(4):895-903.

6. Anand P, Nair HB, Sung B, et al. Design of curcumin-loaded PLGA nanoparticles formulation with enhanced cellular uptake, and increased bioactivity in vitro and superior bioavailability in vivo. Biochem Pharmacol. 2010;79(3):330-338.

7. Dovigo LN, Pavarina AC, Carmello JC, Machado AL, Brunetti IL, Bagnato VS. Susceptibility of clinical isolates of Candida to photodynamic effects of curcumin. Lasers Surg Med. 2011;43(9):927-934.

8. Martins CV, Da Silva DL, Neres AT, et al. Curcumin as a promising antifungal of clinical interest. J Antimicrob Chemother. 2009; 63(2):337-339.

9. Nguyen TNG, Tran PHL, Van Vo T, Van Tran T, Tran TTD. Dissolution enhancement of curcumin by solid dispersion with polyethylene glycol 6000 and hydroxypropyl methylcellulose. In: 5th International Conference on Biomedical Engineering in Vietnam. Cham, Switzerland Springer International Publishing; 2015:298-301.

10. Gosenca M, Bešter-Rogač $M$, Gašperlin M. Lecithin based lamellar liquid crystals as a physiologically acceptable dermal delivery system for ascorbyl palmitate. Eur J Pharm Sci. 2013;50(1):114-122.
11. Calixto G, Bernegossi J, Fonseca-Santos B, Chorilli M. Nanotechnologybased drug delivery systems for treatment of oral cancer: a review. Int J Nanomedicine. 2014:9:3719.

12. Bonifácio BV, da Silva PB, dos Santos Ramos MA, Negri KM, Bauab TM, Chorilli M. Nanotechnology-based drug delivery systems and herbal medicines: a review. Int J Nanomedicine. 2014;9:1-15.

13. Oliveira MB, do Prado AH, Bernegossi J, et al. Topical application of retinyl palmitate-loaded nanotechnology-based drug delivery systems for the treatment of skin aging. Biomed Res Int. 2014; 2014:632570.

14. Bruschi ML, de Freitas O, Lara EH, Panzeri H, Gremião MP, Jones DS. Precursor system of liquid crystalline phase containing propolis microparticles for the treatment of periodontal disease: development and characterization. Drug Dev Ind Pharm. 2008;34(3):267-278.

15. Carvalho FC, Campos ML, Peccinini RG, Gremião MP. Nasal administration of liquid crystal precursor mucoadhesive vehicle as an alternative antiretroviral therapy. Eur J Pharm Biopharm. 2013;84(1): 219-227.

16. Hyde ST. Identification of lyotropic liquid crystalline mesophases. In: Holmberg K, editor. Handbook of Applied Surface and Colloid Chemistry. West Sussex, England: Wiley; 2002:299-332.

17. Rossetti FC, Fantini MC, Carollo AR, Tedesco AC, Bentley MV. Analysis of liquid crystalline nanoparticles by small angle X-ray diffraction: evaluation of drug and pharmaceutical additives influence on the internal structure. J Pharm Sci. 2011;100(7):2849-2857.

18. Gonçalez ML, Corrêa MA, Chorilli M. Skin delivery of kojic acidloaded nanotechnology-based drug delivery systems for the treatment of skin aging. Biomed Res Int. 2013;2013:271276.

19. Carvalho FC, Sarmento VH, Chiavacci LA, Barbi MS, Gremião MP. Development and in vitro evaluation of surfactant systems for controlled release of zidovudine. J Pharm Sci. 2010;99(5):2367-2374.

20. Smart JD, Riley RG, Tsibouklis J, et al. The retention of 14 C-labelled poly (acrylic acids) on gastric and oesophageal mucosa: an in vitro study. Eur J Pharm Sci. 2003;20(1):83-90.

21. Carvalho FC, Calixto G, Hatakeyama IN, Luz GM, Gremião MP, Chorilli M. Rheological, mechanical, and bioadhesive behavior of hydrogels to optimize skin delivery systems. Drug Dev Ind Pharm. 2013; 39(11):1750-1757.

22. Johal HS, Garg T, Rath G, Goyal AK. Advanced topical drug delivery system for the management of vaginal candidiasis. Drug Deliv. 2014:1-14.

23. Dumortier G, Grossiord JL, Agnely F, Chaumeil JC. A Review of poloxamer 407 pharmaceutical and pharmacological characteristics. Pharm Res. 2006;23(12):2709-2728.

24. Carvalho FC, Bruschi ML, Evangelista RC, Gremião MP. Mucoadhesive drug delivery systems. Brazilian J Pharm Sci. 2010;46(1):1-17.

25. Canella KMNC, Garcia RB. Characterization of chitosn by gel permeation chromatography - influence of preparation method and solvent [Caracterização de quitosana por cromatografia de permeação em gel - influência do método de preparação e do solvent]. Quim Nova. 2001;24(1):13-17. Portugese.

26. Owen DH, Katz DF. A vaginal fluid simulant. Contraception. 1999; 59(2):91-95.

27. Vermani K, Garg S, Zaneveld LJ. Assemblies for in vitro measurement of bioadhesive strength and retention characteristics in simulated vaginal environment. Drug Dev Ind Pharm. 2002;28(9):1133-1146.

28. Cevher E, Şensoy D, Zloh M, Mülazımoğlu L. Preparation and characterisation of natamycin: $\gamma$-cyclodextrin inclusion complex and its evaluation in vaginal mucoadhesive formulations. J Pharm Sci. 2008; 97(10):4319-4335.

29. Calixto G, Yoshii AC, Rocha e Silva H, Stringhetti Ferreira Cury B, Chorilli M. Polyacrylic acid polymers hydrogels intended to topical drug delivery: preparation and characterization. Pharm Dev Technol. 2015;20(4):490-496.

30. de Freitas Araújo MG, Pacífico M, Vilegas W, et al. Evaluation of Syngonanthus nitens (Bong.) Ruhl. extract as antifungal and in treatment of vulvovaginal candidiasis. Med Mycol. 2013;51(7):673-682. 
31. Duarte MC, Figueira GM, Sartoratto A, Rehder VL, Delarmelina C. Anti-Candida activity of Brazilian medicinal plants. J Ethnopharmacol. 2005;97(2):305-311.

32. Oliveira MB, Calixto G, Graminha M, Cerecetto H, González M, Chorilli M. Development, characterization, and in vitro biological performance of fluconazole-loaded microemulsions for the topical treatment of cutaneous leishmaniasis. Biomed Res Int. 2015;2015:396894.

33. Prestes PS, Chorilli M, Chiavacci LA, Scarpa MV, Leonardi GR. Physicochemical characterization and rheological behavior evaluation of the liquid crystalline mesophases developed with different silicones. J Dispers Sci Technol. 2009;31(1):117-123.

34. Carvalho FC, Barbi MS, Sarmento VH, Chiavacci LA, Netto FM, Gremião MP. Surfactant systems for nasal zidovudine delivery: structural, rheological and mucoadhesive properties. J Pharm Pharmacol. 2010;62(4):430-439.

35. Takeuchi H, Thongborisute J, Matsui Y, Sugihara H, Yamamoto H, Kawashima Y. Novel mucoadhesion tests for polymers and polymercoated particles to design optimal mucoadhesive drug delivery systems. Adv Drug Deliv Rev. 2005;57(11):1583-1594.
36. Chang JY, Oh YK, Choi HG, Kim YB, Kim CK. Rheological evaluation of thermosensitive and mucoadhesive vaginal gels in physiological conditions. Int J Pharm. 2002;241(1):155-163.

37. Niazy EM. Influence of oleic acid and other permeation promoters on transdermal delivery of dihydroergotamine through rabbit skin. Int J Pharm. 1991;67(1):97-100.

38. Martindale RG, Shikora SA, Nishikawa R, Siepler JK. The metabolic response to stress and alterations in nutrient metabolism. In: Shikora SA, Martindale RG, Steven D, editors. Nutritional Considerations in the Intensive Care Unit-Science, Rationale and Practice. Dubuque, IA: Kendall/Hunt; 2002:11-20.

39. Souza C, Watanabe E, Borgheti-Cardoso LN, Fantini MC, Lara MG. Mucoadhesive system formed by liquid crystals for buccal administration of poly(hexamethylene biguanide) hydrochloride. J Pharm Sci. 2014:1-10.
International Journal of Nanomedicine

\section{Publish your work in this journal}

The International Journal of Nanomedicine is an international, peerreviewed journal focusing on the application of nanotechnology in diagnostics, therapeutics, and drug delivery systems throughout the biomedical field. This journal is indexed on PubMed Central,

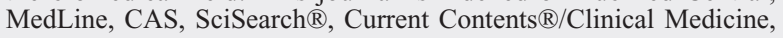

\section{Dovepress}

Journal Citation Reports/Science Edition, EMBase, Scopus and the Elsevier Bibliographic databases. The manuscript management system is completely online and includes a very quick and fair peer-review system, which is all easy to use. Visit http://www.dovepress.com/ testimonials.php to read real quotes from published authors. 